\title{
AN IDENTITY IN THE FREE LIE ALGEBRA
}

\author{
DAVID WIGNER
}

(Communicated by Jonathan Rosenberg)

\begin{abstract}
An identity in the free Lie algebra is shown to imply the theorems
\end{abstract} of Friedrichs and Specht-Wever.

Let $\mathscr{L}(V)$ denote the free Lie algebra on the vector space $V$ over the field $k$. By 'abstract nonsense', we may identify the universal enveloping algebra $U(\mathscr{L}(V))$ with the tensor algebra $\mathscr{T}(V)$ on $V$. The algebra $\mathscr{T}(V)$ possesses a natural grading, $\mathscr{T}(V)=\bigoplus_{0}^{\infty} \mathscr{T}^{n}(V)$. We have $\mathscr{T}^{0}(V)=k$ and $\mathscr{T}^{1}(V)=V$. (We consider $V$ as embedded in $\mathscr{L}(V)$ and $\mathscr{L}(V)$ as embedded in $\mathscr{T}(V)$.) Let $\Delta$ denote the diagonal map of $\mathscr{T}(V)$ and $\mu$ the multiplication. Let $\gamma: \mathscr{T}(V) \rightarrow \mathscr{L}(V)$ be the map defined by the formula $\gamma\left(v_{1} \ldots v_{n}\right)=\left[v_{1}\left[v_{2} \ldots\left[v_{n-1}, v_{n}\right] \ldots\right]\right]$ for $v_{1}, \ldots, v_{n} \in V$. We define $\left.\gamma\right|_{\tau^{0}(V)}=0$ and $\left.\gamma\right|_{\tau^{1}(V)}=\operatorname{Id}_{V}$. If $x \in \mathscr{T}(V)$ and $y \in \bigoplus_{1}^{\infty} \mathscr{T}^{n}(V)$, then $\gamma(x y)=\operatorname{ad}(x)(\gamma(y))$.

Proposition. If $x \in \mathscr{T}^{n}(V)$, then

$$
\mu \circ\left(\gamma \otimes \operatorname{Id}_{\mathscr{T}(V)}\right) \circ \Delta(x)=n x .
$$

Proof. The proof is by induction on $n$, the cases $n=0$ and $n=1$ being clear. Let $x=v_{1} \ldots v_{n}$ with $v_{1}, \ldots, v_{n} \in V$. We may write $\Delta\left(v_{2} \ldots v_{n}\right)=$ $1 \otimes v_{2} \ldots v_{n}+\sum a_{i} \otimes b_{i}$ with each $a_{i} \in \bigoplus_{1}^{\infty} \mathscr{T}^{n}(V)$. The inductive hypothesis gives $\sum \gamma\left(a_{i}\right) \cdot b_{i}=(n-1) v_{2} \ldots v_{n}$. We have

$$
\begin{aligned}
\mu \circ\left(\gamma \otimes \operatorname{Id}_{\mathscr{T}(V)}\right) \circ \Delta\left(v_{1} \ldots v_{n}\right)= & \mu \circ\left(\gamma \otimes \operatorname{Id}_{\mathscr{T}(V)}\right)\left\{( v _ { 1 } \otimes 1 + 1 \otimes v _ { 1 } ) \left(1 \otimes v_{2} \ldots v_{n}\right.\right. \\
& \left.\left.+\sum a_{i} \otimes b_{i}\right)\right\} \\
= & v_{1} \ldots v_{n}+\sum\left\{\operatorname{ad}\left(v_{1}\right)\left(\gamma\left(a_{i}\right)\right) \cdot b_{i}+\gamma\left(a_{i}\right) \cdot v_{1} \cdot b_{i}\right\} \\
= & v_{1} \ldots v_{n}+\sum v_{1} \cdot \gamma\left(a_{i}\right) \cdot b_{i} \\
= & v_{1} \ldots v_{n}+v_{1} \cdot(n-1) v_{2} \ldots v_{n} \\
= & n v_{1} \ldots v_{n} .
\end{aligned}
$$

Received by the editors June 17, 1988, and, in revised form, December 6, 1988.

1980 Mathematics Subject Classification (1985 Revision). Primary 17B35. 
From the proposition we may easily deduce the theorems of Friedrichs ([1, p. 33], [2, p. 170]) and Specht-Wever ([1, p. 34], [2, p. 169]).

Theorem. Suppose that $k$ has characteristic zero. If $x \in \mathscr{T}^{n}(V)$, then the following are equivalent:

(1) $x \in \mathscr{L}(V)$;

(2) $\Delta(x)=1 \otimes x+x \otimes 1$

(3) $\gamma(x)=n x$.

Proof. (1) $\Rightarrow$ (2). Since (2) is true for an element of degree 1, and if $\Delta(x)=$ $1 \otimes x+x \otimes 1$ and $\Delta(y)=1 \otimes y+y \otimes 1$, then $\Delta([x, y])=1 \otimes[x, y]+[x, y] \otimes 1$, we get (2) for elements of $\mathscr{L}(V)$ by induction on the degree.

$(2) \Rightarrow(3)$. From (2) and the proposition we have

$$
\begin{aligned}
n x & =\mu \circ(\gamma \otimes 1) \circ \Delta(x) \\
& =\mu \circ(\gamma \otimes 1)(1 \otimes x+x \otimes 1) \\
& =\gamma(x) .
\end{aligned}
$$

$(3) \Rightarrow(1)$ is clear.

\section{REFERENCES}

1. N. Bourbaki, Groupes et algèbres de Lie, Chapitres 2 et 3, Hermann, Paris 1972.

2. N. Jacobson, Lie algebras, Interscience, New York, 1962.

U.E.R. de Math, Universite Pierre et Marie Curie, 4 Place Jussieu, 75005 Paris, France 